\title{
CN variations in NGC 7006
}

\author{
D. Harbeck ${ }^{1}$, G. H. Smith ${ }^{2}$, and E. K. Grebel ${ }^{1}$ \\ 1 Max-Planck-Institut für Astronomie, Königsstuhl 17, 69117 Heidelberg, Germany \\ ${ }^{2} \mathrm{UCO} /$ Lick Observatory, University of California, CA 95064, USA
}

Received 11 June 2003 / Accepted 29 July 2003

\begin{abstract}
Rotationally induced mixing with subsequent dredge-up of nucleosynthesized material is discussed as a second parameter of the horizontal branch morphology in globular clusters. $\mathrm{CNO}$ abundances have been proposed as tracers of the dredge up of processed material. NGC 7006 is a prominent example of a second parameter GC: Its HB morphology is too red for its metallicity. We present spectroscopic measurements of $\mathrm{CN}$ molecular band strengths $\mathrm{S}(3839)$ and $\mathrm{CH}$ band $\mathrm{CH}(4300)$ strengths for 12 giants in NGC 7006 to test rotationally-driven mixing as a second parameter in this cluster. Our observations reveal (i) a scatter in star-to-star CN absorption strengths with the same amplitude as seen in other GCs of the same metallicity, but different HB morphologies; (ii) a possible continuous distribution of $\mathrm{CN}$ absorption strength with a preference for $\mathrm{CN}$-enriched stars, and (iii) a possible weak radial gradient in the number ratio of $\mathrm{CN}$-strong and $\mathrm{CN}$-weak stars. We argue against the hypothesis that $\mathrm{CN}$-variations are directly correlated with the second parameter effect of the HB morphology. However, the small sample of stars measured in NGC 7006 prevents us from drawing firm conclusions. Finally, we identify one star of our sample as a foreground dwarf carbon star.
\end{abstract}

Key words. galaxy: globular clusters: general - galaxy: globular clusters: individual: NGC 7006 - stars: horizontal-branch

\section{Introduction}

The great ages ( $>10 \mathrm{Gyr})$ of the globular clusters (GCs) in the Milky Way (MW) ensure that only low-mass $\left(M \leq 0.9 M_{\odot}\right)$ stars are present on the main-sequence, while the post mainsequence stars have roughly the same initial mass for a given metallicity. According to the Vogt-Russell theorem, the initial conditions of a star define its subsequent evolution. Among MW GCs of the same metallicity therefore all post mainsequence stars might be expected to evolve in the same way, provided that they are coeval.

Such expectations are not in accord with observations: (i) While stars of a given $\mathrm{GC}$ are homogeneous in the $[\mathrm{Fe} / \mathrm{H}]$ abundance, differences in light elements such as $\mathrm{C}, \mathrm{N}, \mathrm{O}, \mathrm{Na}$, and $\mathrm{Al}$ among GC red giant branch (RGB) stars are a hint of additional processes governing their evolution that are not included in standard stellar models. Alternatively, the assumption of the same initial conditions on the main sequence may be invalid. (ii) MW globular clusters of the same metallicity show very different morphologies of the horizontal branch (the "second parameter effect"). Additional parameters are required for a description of post main-sequence stellar evolution. In this paper we investigate through a study of NGC 7006 whether the second parameter determining the HB morphology might be related to the abundance inhomogeneities among the RGB stars.

Send offprint requests to: D. Harbeck,

e-mail: harbeck@astro.wisc.edu

\subsection{Morphology of the horizontal branch}

The helium core-burning sequence of a GC, i.e., the horizontal branch (HB), is populated by stars of the same initial mass, and typical present-day masses of $M \sim 0.6 M_{\odot}$. The HB morphology can be quantified as follows (Lee et al. 1994):

$\mathrm{HB}=\frac{\# B-\# R}{\# B+\# V+\# R}$,

where $\# B$ and $\# R$ are the number of HB stars bluer or redder than the instability strip, respectively; $\# V$ is the number of variable stars in the instability strip. Predominantly due to differential mass-loss in the RGB phase prior to ignition of helium core-burning, an extended region will be populated along the HB with stars of different envelope masses. Among the MW GCs, there is a general dependence of the HB morphology on cluster metallicity: On average, metal-poor GCs tend to produce HB stars with high effective temperatures (blue HB stars), while metal-rich GCs tend to form red HB stars with lower effective temperatures.

However, it is known that other parameters must affect the evolution of stars to the HB (e.g., Sandage \& Wildey 1960), since there are GCs that do not strictly obey this metallicityHB-morphology relation. For example, some GCs show excessively red HB morphologies at a fixed low metallicity (e.g., NGC 7006, Sandage 1967). Despite numerous studies, the riddle of the second parameter in globular clusters still lacks a comprehensive solution. For instance, age, helium abundance, $\mathrm{CNO}$ abundances, or stellar rotation and subsequently induced 
mixing might affect the HB morphology (as systematically investigated by, e.g., Lee et al. 1994). In general, these parameters regulate the evolution of a main-sequence star along the RGB and onto the HB by changing the mass of the stellar envelope, the mass of the helium burning core, the opacity of the stellar material, or a mixture of these parameters.

Following the arguments of Lee et al. (1994), we briefly summarize how different stellar parameters can change the evolution of stars to the horizontal branch. In this paper we test one particular scenario in which internal abundance spreads in light elements in GCs could be linked to the problem of HB morphology. We concentrate on the outer halo cluster NGC 7006 as it shows a prominent second parameter effect: At a metallicity of $[\mathrm{Fe} / \mathrm{H}]=-1.6$ dex its $\mathrm{HB}$ morphology is redder than that of other GCs of similar [Fe/H], such as M3, M 13, or M 10 (see Table 3). We next discuss how the effects of different parameters on the HB morphology have been explored for NGC 7006.

- Age: Age is a promising candidate to be the second parameter effect: younger GCs would produce redder HBs than older GCs of the same metallicity. For instance, age differences measured with differential age-dating techniques from the main-sequence turn-off (MSTO) might be able to reproduce the differences in the $\mathrm{HB}$ morphologies of the pair NGC 288/NGC 362 at a metallicity of $[\mathrm{Fe} / \mathrm{H}]=$ -1.2 dex, where NGC 288 would be $2 \pm 1$ Gyr older than NGC 362 (Catelan et al. 2001) or for the pair M3/M 13 at a metallicity of $[\mathrm{Fe} / \mathrm{H}]=-1.6 \mathrm{dex}$, where M 13 would be 1.7 \pm 0.7 Gyr older (Rey et al. 2001). Buonanno et al. (1991) found a "normal" age for NGC 7006 from the difference in luminosity of the $\mathrm{HB}$ and the main sequence turn-over. Van den Bergh (1998) and Stetson et al. (1999) found a general trend of outer halo GCs being one to two Gyr younger than the inner halo GCs. However, systematic differences in the $\alpha$-elements might also produce such a distance-age relation. If this general trend applied to NGC 7006, age might be indeed a viable second parameter of the HB morphology. Unfortunately, due to its large distance, no precise relative age-dating of NGC 7006 this cluster is available in the literature.

- CNO abundance: CNO abundances affect both the energy production rate on the main-sequence as well as the stellar opacity; as a net-effect, increased CNO abundances lead to redder horizontal branches. Especially in the case of NGC 7006 the total CNO element abundance has been discussed as a potential second parameter: McClure \& Hesser (1981) found very strong $\mathrm{G}$ band $\mathrm{CH}$ absorption in the RGB stars of this cluster, which might reflect a higher total abundance of CNO elements. Nevertheless, Cohen \& Frogel (1982) could not confirm an overabundance of CNO elements in NGC 7006. These authors found that the infrared $\mathrm{CO}$ absorption band strengths of RGB stars are intermediate between those of M 3 and M 13; both GCs have bluer HB morphologies than NGC 7006. Finally, Wachter et al. (1998) found a depletion of carbon in NGC 7006 giants. This is consistent with the existence of internal $\mathrm{C} \rightarrow \mathrm{N}$ processing where nitrogen is nucleosynthesized by the $\mathrm{CNO}$ cycle in the stellar interior at the cost of carbon and oxygen. Internal mixing processes could bring this material up to the stellar surface. These authors conclude that if the reduced carbon abundance reflects a reduced total $\mathrm{CNO}$ element abundance, then the total amount of $\mathrm{CNO}$ is not likely to be responsible for the second parameter effect in NGC 7006. However, deep mixing can significantly deplete the surface carbon abundance of RGB stars while preserving the total $\mathrm{C}+\mathrm{N}+\mathrm{O}$. Thus, carbon alone might be an improper proxy for the total $\mathrm{CNO}$ abundance of NGC 7006. If CNO enhancement in NGC 7006 would be a second parameter of the $\mathrm{HB}$ morphology, increased $\mathrm{CN}$ and/or CH strength compared to GCs of bluer HB morphology but the same $[\mathrm{Fe} / \mathrm{H}]$ would be expected.

- Rotational mixing as a second parameter: Understanding the effect of rotation on stellar evolution is of particular difficulty, since transport of angular momentum in the interior of stars adds extra challenge. Rotation can affect the later stellar evolution in several ways: rotation would delay the core helium flash, thus leading to higher core masses, resulting in hotter HB stars. Additional mass-loss due to reduced equatorial surface gravity in the later RGB phase would result in lower-mass stellar envelopes, also producing bluer HB stars. Norris (1981) suggested a scenario in which stars with sufficiently high angular momentum would be subject to rotationally-induced interior mixing (e.g., the detailed investigation by Sweigart 1997). Such stars with increased internal mixing could dredge-up CNOcycled, or incompletely $\mathrm{CN}$-cycled or ON-cycled, material, altering the chemical composition of their atmospheres. At the same time, the increased angular momentum could lead to increased core masses as well as increased mass-loss during the RGB evolutionary phase, thus leading to blue $\mathrm{HB}$ stars. Slow rotators on the other hand - appearing $\mathrm{CN}$ poor - would have lower mass loss and would therefore end up as red HB stars. In this scenario a correlation between $C N O$ surface abundances of RGB stars in a $G C$ and the $H B$ morphology would be expected. In the case of M4 Norris (1981) found a similarity between the bimodal distribution functions of color along the $\mathrm{HB}$ and the $\mathrm{CN}$ absorption strengths of RGB stars, which would be consistent with this picture. The scenario in which the angular momentum distribution of stars in a GC would form a second or third parameter of HB morphology seemed promising. However, recent measurements of stellar rotation speeds in M 13 and M 15 (Behr et al. 2000; Behr et al. 2000) identified the red HB stars as having higher mean rotational speeds (as found from the distribution of $v \sin i$ ) than the blue HB stars. In the rotational mixing scenario the opposite distribution would be expected. These observations could still fit a rotational second parameter scenario if increased mass-loss due to increased rotation speed would also imply increased loss of angular momentum, e.g., via stellar winds.

Rotational mixing as a second parameter leads one to expect a relation between horizontal branch morphology and the distributions of the CNO element abundances among GC red giants. We use spectroscopic measurements of $\mathrm{CN}$ and $\mathrm{CH}$ bands of RGB stars as a tool to trace the dredge-up of material that 
has been nucleosynthesized through the CNO-cycle of hydrogen burning. As nitrogen is the minor component in stellar atmospheres compared to $\mathrm{C}$, an enrichment of $\mathrm{N}$ at the cost of $\mathrm{C}$ by the CNO-cycle will result in enhanced molecular $\mathrm{CN}$ formation.

We aim to test the rotational mixing hypothesis by comparing both the range of $\mathrm{CN}$ strengths exhibited by RGB stars, and the relative fraction of $\mathrm{CN}$-rich and $\mathrm{CN}$-poor stars, in NGC 7006 and a set of other GCs having metallicities of $[\mathrm{Fe} / \mathrm{H}] \sim-1.6$ dex but different $\mathrm{HB}$ morphologies. For some of these GCs, measurements of $\mathrm{CN}$ absorption strength are available in the literature. In the scenario of deep mixing one would expect, with metallicity as a fixed parameter, a correlation between the HB morphology of a globular cluster and the star-to-star $\mathrm{CN}$ abundance variations within a cluster. Besides the strong "global" second parameter at work in NGC 7006, Buonanno et al. (1991) found evidence for an "internal" second parameter effect within it: The morphology of the HB becomes bluer with decreasing radial distance. Unless one accepts the unlikely scenario of an age-spread of order of a few billion years within a globular cluster, such an internal second parameter effect provides evidence of additional third parameters other than age, such as environment. It will be interesting to test if this internal HB gradient is also reflected by a gradient in the $\mathrm{CN}$ band strengths. We note that such "internal" 2 nd parameter effects have also been observed in the old field populations of dwarf spheroidal galaxies (Hurley-Keller et al. 1999; Harbeck et al. 2001) and appear there correlated with the RGB color.

\subsection{New $\mathrm{CN}$ and $\mathrm{CH}$ spectroscopy of NGC 7006}

Although NGC7006 is an extreme example of the secondparameter effect in GCs, it is poorly studied. Only a few measurements of $\mathrm{CN}$ absorption strength are available in the literature. The major difficulty of this object is its large distance $(57.4 \mathrm{kpc})$, and only the tip of the RGB has been explored spectroscopically so far. For example, from high-resolution Keck spectra of six stars in NGC 7006 Kraft et al. (1998) detected a $\mathrm{Mg}-\mathrm{Al}$ anti-correlation, which is a strong indication of material nucleosynthesized by hydrogen-shell burning. A common tool for investigating star-to-star inhomogeneities in the CNO elements is spectroscopy of the $3883 \AA \mathrm{CN}$ and $4300 \AA$ $\mathrm{CH}$ molecular absorption bands, which does not require the high resolution needed for atomic absorption line studies. The reduced telescope time required has to be traded off against the loss of detailed information on individual elements. In this paper we report measurements of an index denoted S(3839) for a sample of red giants in NGC 7006. This index has been used in a number of $\mathrm{CN}$ studies. We compare the results with those for other GCs of similar metallicity.

\section{Data and reduction}

During several observing campaigns in the years 1996, 1997, and 2000, spectra of 12 stars among the bright end of the RGB of NGC 7006 were observed with the KAST spectrograph on the Lick $3 \mathrm{~m}$ Shane telescope. The grism No. 1 was used,
Table 1. Logbook of observations.

\begin{tabular}{lll}
\hline \hline Star-ID & Date & Exposure time \\
\hline II-18 & $19 / 09 / 96$ & $3 \times 2700 \mathrm{~s}$ \\
II-46 & $19 / 09 / 96$ & $3 \times 2700 \mathrm{~s}$ \\
V-19 & $20 / 09 / 96$ & $3 \times 2700 \mathrm{~s}$ \\
V-54 & $20 / 09 / 96$ & $1 \times 2700 \mathrm{~s}$ \\
II-103 & $02 / 08 / 97$ & $2 \times 3600 \mathrm{~s}$ \\
II-89 & $02 / 08 / 97$ & $2 \times 3600 \mathrm{~s}+1 \times 2700 \mathrm{~s}$ \\
III-1 & $03 / 08 / 97$ & $1 \times 3600 \mathrm{~s}+1 \times 1800 \mathrm{~s}$ \\
II-85 & $03 / 08 / 97$ & $2 \times 3600 \mathrm{~s}+1 \times 2700 \mathrm{~s}$ \\
II-4 & $25 / 08 / 97$ & $3 \times 3600 \mathrm{~s}$ \\
III-46 & $25 / 08 / 97$ & $2 \times 3600 \mathrm{~s}+1 \times 2400 \mathrm{~s}$ \\
III-33 & $04 / 07 / 00$ & $2 \times 3600 \mathrm{~s}+2 \times 2700 \mathrm{~s}$ \\
III-40 & $05 / 07 / 00$ & $3 \times 3600 \mathrm{~s}+1 \times 2700 \mathrm{~s}$ \\
\hline
\end{tabular}

resulting in a wavelength coverage from $3200 \AA$ to $6500 \AA$ at a dispersion of $2.8 \AA /$ pixel. A $1200 \times 400$ Reticon CCD with a pixel size of $27 \mu \mathrm{m}$ and $6 \mathrm{e}^{-}$readout noise was used as detector. Per observing run typically 20 bias observations and 20 dome flat fields were taken. $\mathrm{HeHgCd}$ arc lamp exposures for wavelength calibration and flux standard star spectra were obtained. A logbook of the observations is given in Table 1; the identification of the observed stars is the same as in Sandage (1967).

The data were reduced separately for each observing campaign using IRAF $^{1}$ data reduction routines. The KAST CCD readout system automatically subtracts a bias level from the images estimated from the overscan line. We found that the illumination level on the chip affects the estimate for the overscan level (in particular for highly illuminated flat fields). This effect leads to an overestimation of the overscan level in some images. Since the subtracted overscan is stored in the last column of each KAST CCD image, we added back the overscan level. All bias frames were combined and this masterbias was subtracted from all frames. In a subsequent step the flat fields were combined. A polynomial of 25 th order in the dispersion direction was used to eliminate both spectral sensitivity and the energy distribution of the quartz lamp from the flat field. The normalized flat field was used to flatten all data.

The long exposure spectra of NGC 7006 are affected by cosmic ray hits. We found that the best way to remove them was to use the lacos_spec task (van Dokkum 2001). Most of the stars were observed several times, and we coadded the available CCD frames to a final deep, almost cosmic-ray-free image. The spectra were extracted with the specred package in IRAF. From the typically five flux standard stars observed per campaign the spectral sensitivity function was determined and used to flux-calibrate the stellar spectra. Note that the spectra are only calibrated with respect to relative spectral sensitivity, not to absolute flux.

${ }^{1}$ IRAF is distributed by the National Optical Astronomy Observatories, which are operated by the Association of Universities for Research in Astronomy, Inc., under cooperative agreement with the National Science Foundation. 


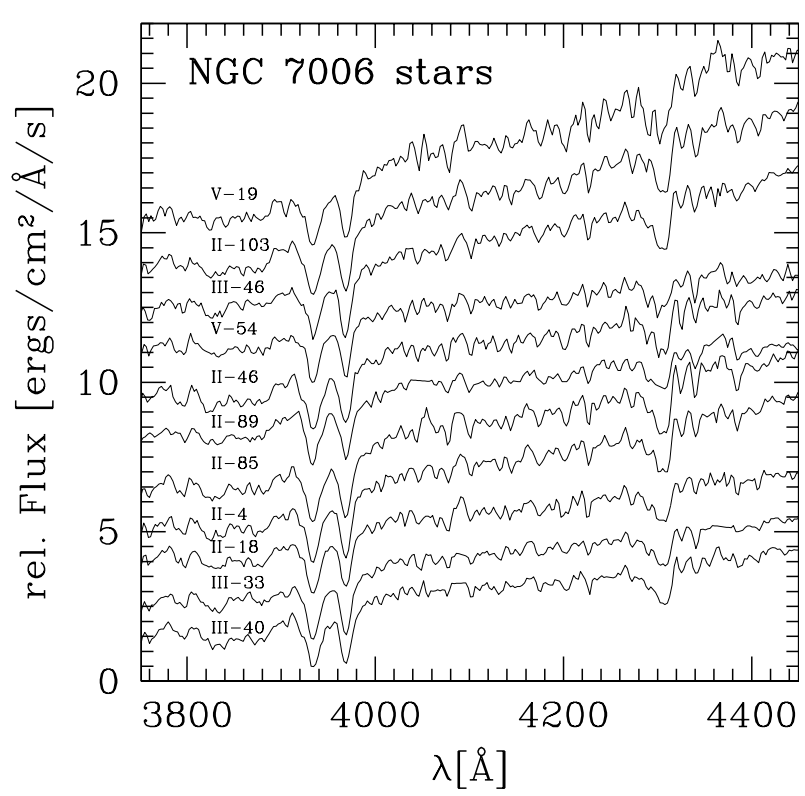

Fig. 1. Spectra of all stars in our sample; the peculiar star III-1 is excluded. The spectra are normalized by their flux at $4200 \AA$, and they are shifted vertically for clarity.

For each spectrum we measured the centers of the $\mathrm{Ca}$ II $\mathrm{H}+\mathrm{K}$ lines with the rvidlines task in the rv package under IRAF to determine the radial velocities of the stars. Since we want to measure spectral indices in the rest frame wavelength, we corrected the spectra for the measured Doppler shift. The final extracted spectra of all stars are plotted in Fig. 1.

Additionally we measured relative radial velocities via cross-correlation against the star II-4 using the fxcor task in the IRAF rv package. We found a nearly Gaussian distribution of relative velocities with a dispersion of $\sim 50 \mathrm{~km} \mathrm{~s}^{-1}$, which reflects the uncertainty in the determination of the radial velocity at the given spectral resolution rather than the internal dispersion of NGC 7006. But there is one significant outlier: The star III-1 was found to have a relative radial velocity of $+245 \mathrm{~km} \mathrm{~s}^{-1}$ compared to II- -4 . We conclude that all stars in our sample but III-1 are members of NGC 7006. The spectrum of III-1 is peculiar and dominated by prominent molecular bands (see Fig. 2). According to its features (strong $\mathrm{C}_{2}$ and $\mathrm{CH}$ absorption bands and strong Mgb lines) we identify this star as a foreground dwarf carbon star.

\subsection{Spectral indices}

From the spectral energy distribution we measured the strength of absorption by the $\mathrm{CN}$ molecule at the $3883 \AA$ band. The CH absorption strength was measured from the $4300 \AA$ $G$-band. The spectral indices $\mathrm{S}(3839)$ and $\mathrm{CH}(4300)$ measure the flux depression in the molecular bands relative to a nearby comparison passband. The indices are defined according to Norris et al. (1981), but we modified the definition of the CH index comparison passband to avoid strong sodium sky emission lines. The $\mathrm{CN}$ index definition remains unchanged, allowing a direct

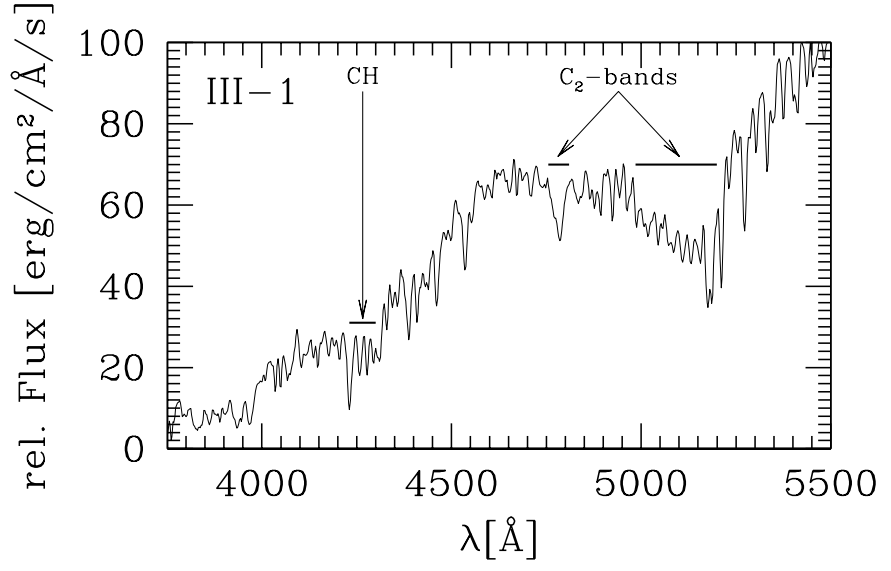

Fig. 2. Spectrum of the peculiar star III-1, which is probably a foreground dwarf carbon star. Note the strong absorption bands due to $\mathrm{CH}$ and $\mathrm{C}_{2}$.

comparison to results of other studies:

$$
\begin{aligned}
& \mathrm{S}(3839)=-2.5 \log \left(\frac{\sum_{3846}^{3883} F_{\lambda}}{\sum_{3883}^{3916} F_{\lambda}}\right) \\
& \mathrm{CH}(4300)=-2.5 \log \left(\frac{\sum_{4280}^{4320} F_{\lambda}}{\sum_{4250}^{4280} F_{\lambda}+\sum_{4320}^{4340} F_{\lambda}}\right) .
\end{aligned}
$$

Larger index values correspond to increased molecular band absorption. An additional spectral index S(4241) to measure $\mathrm{CN}$ absorption in the molecular band at $4215 \AA$ is not considered here, since the absorption in this band is very low for metal-poor stars. Most of the stars were observed several times. For two representative stars we tested how well the individual measurements of the S(3839) index would reproduce the measurement on the combined spectrum. The typical scatter in S(3839) among the short exposures is of or$\operatorname{der} \sigma \mathrm{S}(3839)= \pm 0.03 \mathrm{mag}$. To account for different exposure times of the stars, we determine the uncertainty of the $\mathrm{CN}$ and $\mathrm{CH}$ indices based on the Poisson noise of the number counts within the index passbands, resulting in error estimates that are of similar magnitude in both indices. The measured indices as well as photometry from Sandage (1967) are listed in Table 2. In the following we will transform the apparent magnitudes of NGC 7006 stars to absolute magnitudes using a distance modulus of $(m-M)_{V}=18.24$ and correcting for a reddening of $E(B-V)=0.05$ (Harris 1996).

\section{Results}

The formation of $\mathrm{CN}$ and $\mathrm{CH}$ molecules is affected by temperature as well as by gravity effects. Since our sample spans only a small range in color (temperature) and luminosity, this dependence of the molecule formation cannot easily be determined. We compare the NGC $7006 \mathrm{~S}(3839)$ strengths to those of the well studied GCs M 3 and M 10, which have the same metallicity as NGC 7006. Smith (2002b) homogenized CN S(3839) measurements of stars in M 3 from numerous sources in the literature. For M 10, we use the measurements of Smith \& Fullbright (1997). One result of the Smith (2002b) 
Table 2. Parameters of program stars.

\begin{tabular}{lccccc}
\hline \hline Star-ID & $V$ & $(B-V)$ & $\mathrm{S}(3839)$ & $\mathrm{CH}(4300)$ & {$[\mathrm{C} / \mathrm{Fe}]^{1}$} \\
\hline II-103 & 16.23 & 1.41 & $0.302 \pm 0.06$ & $1.367 \pm 0.03$ & \\
II-18 & 16.32 & 1.33 & $0.149 \pm 0.07$ & $1.338 \pm 0.04$ & \\
II-4 & 16.62 & 1.21 & $0.139 \pm 0.07$ & $1.404 \pm 0.04$ & -0.82 \\
II-46 & 16.38 & 1.38 & $0.239 \pm 0.07$ & $1.374 \pm 0.04$ & \\
II-85 & 16.60 & 1.20 & $0.115 \pm 0.07$ & $1.410 \pm 0.04$ & \\
II-89 & 16.53 & 1.21 & $0.243 \pm 0.07$ & $1.324 \pm 0.04$ & \\
III-1 & 16.44 & 1.37 & $-0.017 \pm 0.07$ & $1.428 \pm 0.04$ & \\
III-33 & 16.77 & 1.10 & $-0.038 \pm 0.07$ & $1.341 \pm 0.05$ & -0.97 \\
III-40 & 16.96 & 1.12 & $0.208 \pm 0.09$ & $1.404 \pm 0.06$ & -0.75 \\
III-46 & 16.78 & 1.24 & $0.042 \pm 0.06$ & $1.409 \pm 0.04$ & \\
V-19 & 15.50 & 1.72 & $0.120 \pm 0.07$ & $1.367 \pm 0.04$ & \\
V-54 & 15.85 & 1.35 & $0.066 \pm 0.05$ & $1.289 \pm 0.03$ & \\
\hline
\end{tabular}

${ }^{1}$ Adopted from Friel et al. (1982).

study is a good description of the dependence of the $\mathrm{CN}$ absorption strength on the luminosity/temperature for stars on the upper RGB. A prominent feature is the decrease of $\mathrm{CN}$ absorption strengths for stars brighter than $M_{V} \leq-1.8$ mag. In Fig. 3 we compare our measurements of the $3883 \AA \mathrm{CN}$ band for NGC 7006 (filled circles) with the literature data for M 3 (tripods) and M 10 (open squares). The temperature effect on the $\mathrm{CN}$ formation efficiency is clearly visible in the M 3 sample; with increasing luminosity (=decreasing surface temperature) the formation of $\mathrm{CN}$ becomes more efficient. At the turnover at $M_{V}=-1.8 \mathrm{mag}$ the $\mathrm{CN}$ formation is reduced for increasing luminosities as described by Smith (2002b). We observed only the brightest stars in NGC 7006, in particular all stars are at the luminosity of or brighter than the $\mathrm{CN}$-turnover luminosity. Two bright (and red) stars in our sample are identified variable stars; at their location in the color-magnitude diagram the risk of AGB star contamination is high, and for a comparison between M3, M 10, and NGC 7006 we favor concentrating on the stars closer to the $\mathrm{CN}$-index turn-over region.

\subsection{Range of $\mathrm{CN}$ absorption strengths}

The range of $\mathrm{CN}$ absorption strengths at a given magnitude in NGC 7006 (see Fig. 3) is comparable, within the errorbars, to the range covered by the globular clusters M3 and M 10. Both comparison clusters have metallicities comparable to NGC 7006 (see Table 3), but bluer HB morphologies (HB-values of 0.08 and 0.98 , respectively). There is no clear indication in NGC 7006 of a bimodal distribution of CN absorption strengths as is observed in M3, M 10, and in other globular clusters. A continuous distribution of S(3839) absorption strengths is possible for NGC 7006, although the small size of our star sample prohibits a unique determination of the $\mathrm{CN}$ distribution function.

In the scenario of rotational mixing as the second parameter we would expect stars in GCs with blue HB morphologies to have, on average, higher angular momentum than stars in

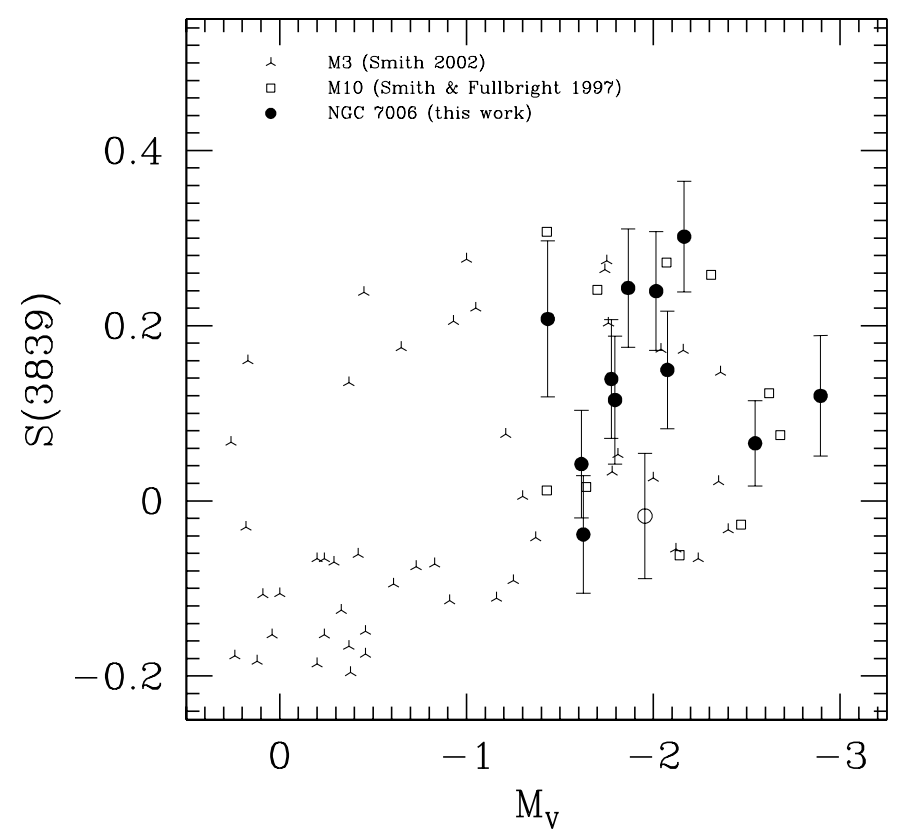

Fig. 3. Comparison of S(3839) absorption strength in NGC7006 (filled circles), M 3 (stars) and M 10 (open boxes). In the magnitude range covered by our observations the scatter in the NGC $7006 \mathrm{CN}$ absorption strength compares well to those in M 3 and M 10. There is no clear bimodal signature in NGC 7006 as is visible in M3. The foreground dC star III-1 is plotted with an open circle.

Table 3. $[\mathrm{Fe} / \mathrm{H}]$ and HB morphologies from Harris (1996); $r$ is the number ratio $r=\frac{\# \mathrm{CN}-\text { strong }}{\# \mathrm{CN}-\text { weak }}$. References: 1 - Briley \& Smith (1993); 2 - Smith \& Fullbright (1997), 3 - Norris (1987), 4 - Smith (2002b), 5 - Smith \& Norris (1982); 6 - Langer et al. (1992), 7 - Norris \& Freeman (1983), 8 - Norris et al. (1981); 9 - Smith \& Bell (1986), 10 - Smith \& Mateo (1990).

\begin{tabular}{lllll}
\hline \hline Cluster & {$[\mathrm{Fe} / \mathrm{H}]$} & $\mathrm{HB}$ & $\mathrm{r}$ & Sources \\
\hline M 10 & -1.52 & 0.98 & 0.71 & 1,2 \\
M 13 & -1.54 & 0.97 & 3.2 & 3 \\
M 3 & -1.57 & 0.08 & 0.42 & 4 \\
NGC 3201 & -1.58 & 0.08 & 1.00 & 5 \\
NGC 1904 & -1.57 & 0.89 & 2.60 & 6 \\
M 22 & -1.64 & 0.91 & 0.42 & 7 \\
NGC 6752 & -1.56 & 1.00 & 0.95 & 8 \\
NGC 6934 & -1.54 & 0.25 & 0.58 & 9 \\
M 2 & -1.62 & 0.96 & 3.5 & 10 \\
NGC 7006 & -1.63 & -0.28 & $(2-3.5)$ & this work \\
\hline
\end{tabular}

GCs with red HB morphologies. We consequently expect that stars in NGC 7006 should be slower rotators than those in M 3 or M 10. Under this assumption, stars in NGC 7006 would experience less rotationally induced mixing, and less nucleosynthesized material should become dredged-up to the stellar envelope. If rotation were primarily responsible for determining both $\mathrm{HB}$ morphology and $\mathrm{CN}$ inhomogeneities, a smaller range in $\mathrm{CN}$ absorption strength and/or a preference for stars with low $\mathrm{CN}$ abundances would be expected in NGC 7006. Our observation of a range of $\mathrm{S}(3839)$ values in NGC 7006 that is comparable to those seen in GCs with bluer HB morphology contradicts 


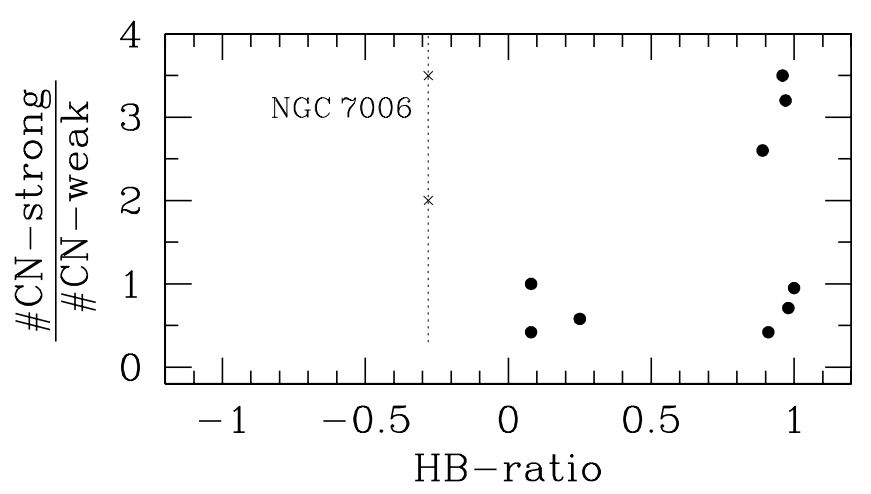

Fig. 4. Comparison of the $\mathrm{HB}$ morphology and the content of $\mathrm{CN}$ enhanced stars in GCs. We plot the HB morphology index $=\frac{\# B-\# R}{\# B+\# V+\# R}$ vs. the number ratio $r$ between $\mathrm{CN}$-enhanced and $\mathrm{CN}$-weak stars. The crosses represent two different estimates of $r$ in NGC 7006 (see text). The dotted line indicates the range $r$ in NGC 7006 consistent with the uncertainties of the number counts.

- within the limitations of the small sample - the predictions of this scenario.

\subsection{Ratio of $\mathrm{CN}$-strong/CN-weak stars}

We cannot identify a correlation between the range of $\mathrm{CN}$ absorption strengths in NGC 7006, M3, and M 10 and the HB morphology. In a second attempt we investigate the ratio

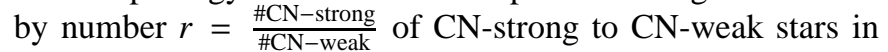
GCs with metallicities comparable to NGC 7006: In the scenario of rotationally induced mixing the distribution of $\mathrm{CN}$ absorption strengths should be weighted to $\mathrm{CN}$-poor stars in GCs with low mean stellar angular momentum (and relatively red HB morphologies), while $\mathrm{CN}$-strong stars should be preferred in GCs with high mean stellar angular momentum (and bluer HB morphologies).

For GCs with bimodal distributions of $\mathrm{CN}$ absorption strengths a clear classification into $\mathrm{CN}$-strong and $\mathrm{CN}$-weak stars is possible. We collected values of $r$ for GCs with metallicities comparable to NGC 7006 where data are available from the literature in Table 3. The metallicities and HB morphologies are taken from Harris (1996). In NGC 7006 the classification of $\mathrm{CN}$ absorption strengths is ambiguous: if we consider all NGC 7006 stars with magnitudes $M_{V} \geq-2.4$ and $\mathrm{S}(3839) \geq 0.1$ as $\mathrm{CN}$-strong (see Fig. 3), we obtain a number ratio of $r=\frac{7}{2}=3.5$. Ignoring stars with intermediate $\mathrm{S}(3839)$ absorption strengths $(0.1 \leq \mathrm{S}(3839) \leq 0.18)$, results in $r=\frac{4}{2}=2$.

Figure 4 shows the $\mathrm{HB}$ index vs. the $\mathrm{CN}$-ratio $r$ for the sample of GCs with metallicities comparable to NGC 7006. Due to the ambiguity of $r$ in NGC 7006 we refrain from boldly plotting it in this diagram. Instead, we indicate the range of $r$ that is consistent with our data sample. In this sample, no clear correlation between the HB morphology and the ratio of $\mathrm{CN}$-strong to $\mathrm{CN}$-weak stars is apparent, as also found in a similar study by Smith (2002a). M 2 and NGC 1904 fulfill the expectation of the rotation plus mixing scenario that GCs with extremely blue HB morphology should have more $\mathrm{CN}$-strong stars than GCs with a redder $\mathrm{HB}$ morphology. But at the same time there are

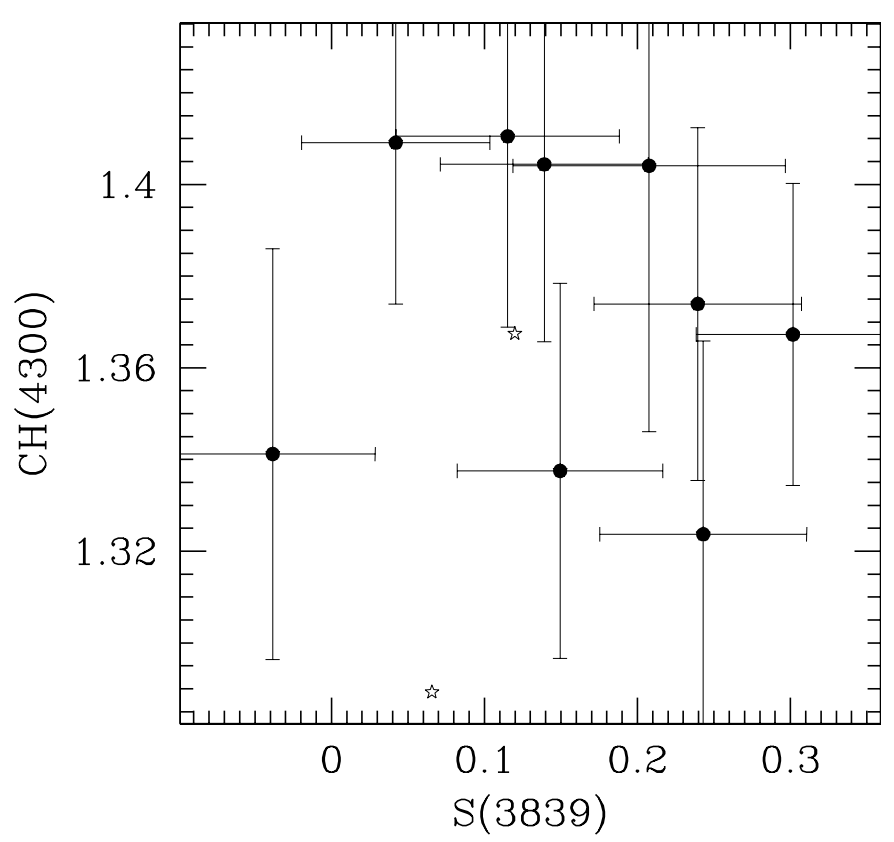

Fig. 5. Comparison of the $\mathrm{CN}$ and $\mathrm{CH}$ absorption strengths in NGC 7006. Stars with $M_{V} \geq-2.3$ mag are plotted with filled circles. The two brightest giants in the sample are plotted with open stars.

GCs with very blue HB morphologies and low number ratios $r$ comparable to GCs with normal HB morphologies. NGC 7006 seems to have too large a ratio $r$ to support the scenario of an $r$-HB-type correlation. If the distribution of angular momentum among stars in a GC is correlated with the fraction of $\mathrm{CN}$ enhanced stars, stellar rotation cannot be identified as a second parameter of the HB morphology, at least for our sample of GCs with metallicities of $[\mathrm{Fe} / \mathrm{H}] \sim-1.6 \mathrm{dex}$. We would like to point out that a correlation between the distribution of $\mathrm{CN}$ absorption strengths and stellar rotation in GCs has still to be proven. Since the initial distribution of angular momentum can only be measured on the main-sequence of GCs with high-resolution spectroscopy, limitations in telescope size and instrumentation have not permitted such studies so far. While main-sequence stars in GCs such as M3 and M 13 are now in reach of large telescopes, the distant cluster NGC 7006 is still too far away for efficient studies of such stars.

\subsection{An anti-correlation between $\mathrm{CN}$ and $\mathrm{CH}$ ?}

For a variety of GCs with $\mathrm{CN}$ abundance inhomogeneities an anti-correlation between the $\mathrm{CN}$ band and $\mathrm{CH} G$-band absorption strengths has been detected, e.g., in M 2, M 3, M 10, M 13, and NGC 6752. Such a CN-CH anti-correlation is expected if $\mathrm{CN}$ variations are produced by nucleosynthesis of material in the CNO cycle. Nitrogen would be enriched at the cost of carbon and oxygen. The $\mathrm{N}$-enhancement will lead to $\mathrm{CN}$-strong stars, while the reduced carbon content in such stars would suppress $\mathrm{CH}$ molecule formation. In Fig. 5 we plot the $\mathrm{S}(3839)$ vs. $\mathrm{CH}(4300)$ spectral index for stars in the narrow luminosity range with $M_{V} \geq-2.3$ mag. The impact of temperature effects on the molecule formation is therefore expected to be small. The uncertainties in the $\mathrm{CH}$ indices, coupled with an apparently 


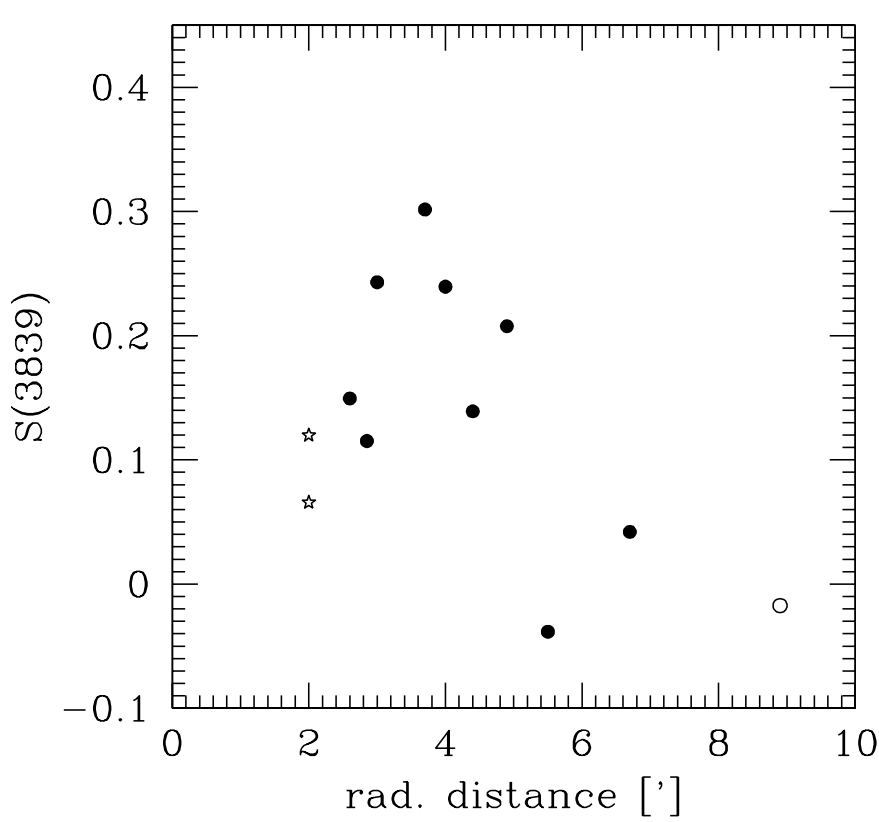

Fig. 6. A plot of the CN absorption strength of NGC 7006 giants vs. projected distance to the cluster center. Stars with strong $\mathrm{CN}$ bands are found at small radial distances, while the two $\mathrm{CN}$-weak stars have large radial distances. Note that there are no $\mathrm{CN}$-weak stars with a radial distance $\leq 5^{\prime}$. Only stars with $M_{V} \geq-2.3$ are plotted with filled circles. The open circle indicates the location of the field star III-1. The two brightest giants are plotted with open stars.

small range in $\mathrm{CH}(4300)$ values among our NGC 7006 giants, do not allow a quantification of the true scatter in $\mathrm{CH}$ absorption strength.

Three of our stars (III-40, II-4, and III-33) are in common with the sample investigated by Friel et al. (1982), who determined carbon abundances for these stars from low-resolution spectroscopy (included in Table 2). We find a reasonable correlation between our measured $\mathrm{CH}(4300)$ band strengths and the Friel et al. (1982) carbon abundance: Star III-33 - having the lowest $[\mathrm{C} / \mathrm{Fe}]$ abundance among the three stars - also has the lowest measured $\mathrm{CH}$ absorption band strength. On the other hand, star III-33 also has the lowest S(3839) absorption strength, which also appears to be consistent with Fig. 5a of Friel et al. (1982). We conclude that there is no evidence of a $\mathrm{CN}-\mathrm{CH}$ anti-correlation from Fig. 5, although it might be hidden in the observational uncertainties.

\subsection{Radial distribution of $\mathrm{CN}$ strengths}

NGC 7006 has been reported to show - besides its global second parameter effect - an internal "second" parameter effect. The morphology of the HB changes with the radial distance to the cluster's center (Buonanno et al. 1991): The HB morphology appears bluer in the central parts than in the outskirts. It appears therefore interesting to see if a similar trend can be found for the distribution of $\mathrm{CN}$ absorption strength. Our sample of eleven NGC 7006 stars lacks the statistical significance of a large survey. Nevertheless, we investigate the radial distribution of $\mathrm{CN}$-weak and $\mathrm{CN}$-strong stars in our sample. In Fig. 6 we plot the $\mathrm{CN}$ index $\mathrm{S}(3839)$ vs. the projected radial distance from the cluster center on the sky. Surprisingly, all $\mathrm{CN}$-enhanced stars have distances smaller than 4.5', while the two $\mathrm{CN}$-weakest stars have larger distances. A radial distance of $4.5^{\prime}$ corresponds to approximately 0.7 tidal radii $\left(r_{\mathrm{t}}=6.34^{\prime}\right.$; Harris 1996). The lack of $\mathrm{CN}$-strong stars at large radial distances is an uncertain result due to the small number of observed stars; the absence of CN-poor stars in the cluster's inner part is worth noting. We test the significance of a difference in the radial distributions of $\mathrm{CN}$-strong $(\mathrm{S}(3839) \geq 0.1)$ and CN-weak $(\mathrm{S}(3839) \leq 0.1)$ stars with a Kolmogorov-Smirnov test. This test results in a $0.6 \%$ probability that $\mathrm{CN}$-strong and $\mathrm{CN}$-weak stars follow the same radial distribution. It is worth mentioning here that Buonanno et al. (1991) found the radial gradient in HB morphologies on a scale of order of $70^{\prime \prime}$, while we find a lack of CN-strong stars on radial scales of order $4^{\prime}$. A detailed study of $\mathrm{CN}$ - especially in the central parts - appears desirable.

A radial gradient in the distribution of $\mathrm{CN}$ strengths has been found in the globular cluster 47 Tuc (Norris \& Freeman 1979). In this cluster, CN-strong stars are more centrally concentrated than $\mathrm{CN}$-weak stars. Briley (1997) pointed out that there are no $\mathrm{CN}$-weak stars in the very center of 47 Tuc. The same situation might be seen in NGC 7006. Although the gradient in NGC 7006 has to be proven by a larger sample, it would not be without precedent.

If this gradient in $\mathrm{CN}$ absorption strength is indeed real, its implication is not unique. In the scenario of rotationally induced mixing this gradient would reflect a gradient in the angular momentum distribution of the stars, where stars in the inner part of NGC 7006 would have higher angular momentum. Accordingly, the central stars would experience enhanced internal mixing and enhanced mass loss. It is not clear if the distribution of angular momentum dates to the star formation epoch of NGC 7006, or if close stellar encounters with transfer of angular momentum lead to a spin-up of those stars orbiting preferentially within the inner parts of the GC.

In other scenarios, such as accretion of CNO processed stellar winds (e.g., investigated in detail by Thoul et al. 2002), the gradient in $\mathrm{CN}$ strengths would reflect different efficiencies of the accretion process. Stellar winds may settle at the center of a GC; if so, stars with orbits restricted to the central regions would accrete more material than stars on more extended orbits. Thus, the central concentration of $\mathrm{CN}$-enriched stars could be consistent with the accretion scenario. A more detailed investigation of the suggested gradient in $\mathrm{CN}$ absorption strengths in NGC 7006 appears desirable.

\subsubsection{Sodium, oxygen, and CN among NGC 7006 giants}

In GCs with $\mathrm{CN}$ abundance variations, a positive correlation between the sodium abundance and $\mathrm{CN}$ is often found. At the same time, $\mathrm{CN}$ and the oxygen abundance tend to be anticorrelated (e.g., the review by Kraft 1994). An oxygen-CN anti-correlation is a natural consequence of CNO-processing. The situation is less clear in the case of the sodium-CN correlation: initial interpretation of this correlation was that 

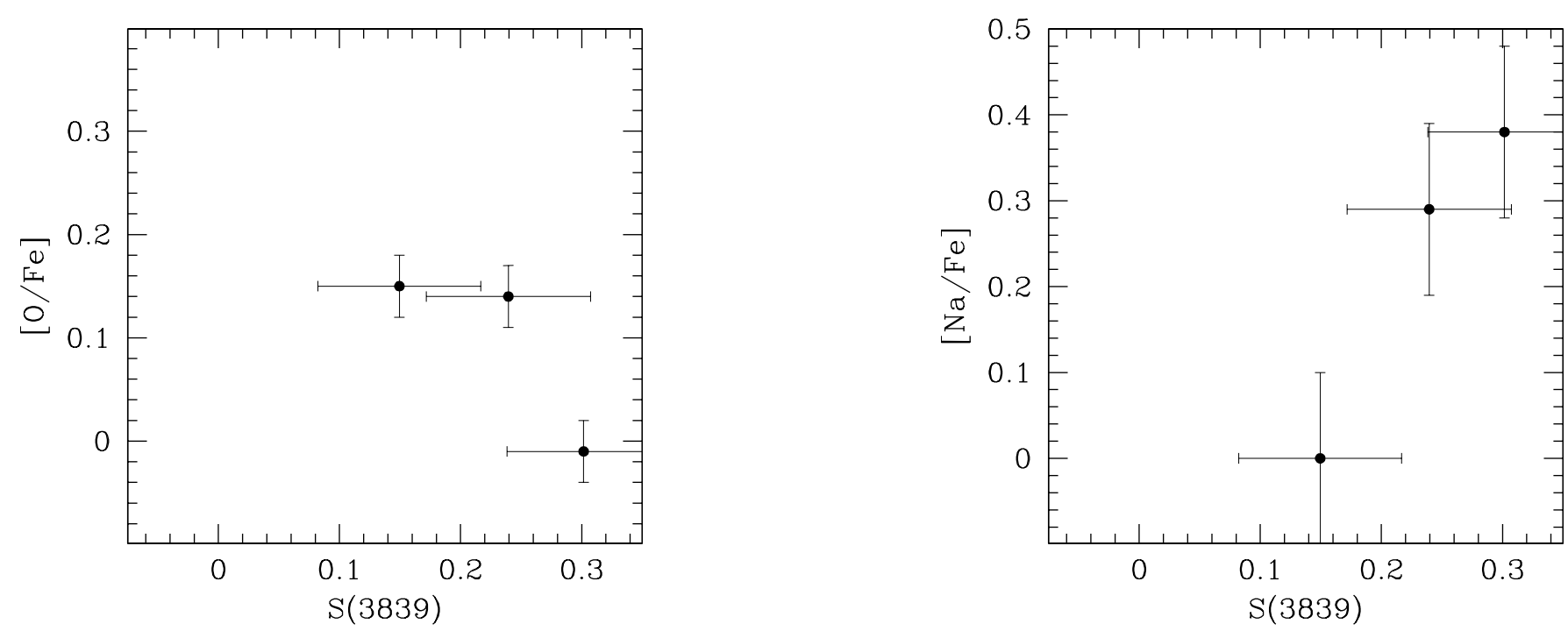

Fig. 7. Comparison of CN S(3839) band strength with oxygen (left) and sodium (right) abundance measurements for stars in common with Kraft et al. (1998). Within the strong limitation of the dataset, an oxygen-CN anti-correlation and a positive sodium-CN correlation is consistent with the observations.

material in $\mathrm{CN}$-strong GC red giants had been nucleosynthesized in more massive stars, whose stellar ejecta were accreted by those stars seen at the present time. However, a neon $\rightarrow$ sodium proton capture chain was identified as a possible mechanism to allow internal sodium enhancement even in low-mass stars (Denisenkov \& Denisenkova 1990; Langer et al. 1993; Cavallo et al. 1996, 1998). If this process is indeed present in RGB stars, the internal mixing scenario would still be consistent with a sodium-CN correlation as has been argued by a number of authors (e.g., Weiss et al. 2000).

Does NGC 7006 obey the general correlations between $\mathrm{CN}$, sodium, and oxygen observed in other GCs? In Fig. 7 we compare the $\mathrm{CN}$ absorption strengths measured in this study to the sodium and oxygen abundances measured by Kraft et al. (1998) from high-resolution spectroscopy obtained at the Keck telescope. There are five stars in common with this study, including the two peculiar variable stars which are excluded from this comparison. The three remaining stars with measured oxygen and sodium abundances are III-33, III-48, and III-46. All stars are intermediate or strong in $\mathrm{CN}$; no $\mathrm{CN}$-poor stars are included in this common sample. Although lacking strong significance, a $\mathrm{CN}$-oxygen anti-correlation as well as a $\mathrm{CN}$-sodium positive correlation is suggested.

\section{Summary}

Based on single-slit spectroscopy at the $3 \mathrm{~m}$ Shane telescope obtained in seven nights of observing time we have measured $\mathrm{CN}$ and $\mathrm{CH}$ molecule absorption strengths for twelve stars in NGC 7006. We discussed the variations in $\mathrm{CN}$ and $\mathrm{CH}$ in the context of the second parameter effect of horizontal branches in GCs. Within the limitations of this modest set of CN observations, we found indications of (i) a continuous distribution of CN S(3839) index values, (ii) a range in $\mathrm{CN}$ absorption strength among RGB stars comparable to the $\mathrm{CN}$ spread present in the GCs M 3 and M 10, and (iii) a larger fraction of RGB stars seem to be CN-enriched in NGC 7006 than in the two comparison clusters. A radial gradient in the $\mathrm{CN}$ enrichment of stars in NGC 7006 is suggested.

At a metallicity of $[\mathrm{Fe} / \mathrm{H}] \sim-1.6 \mathrm{dex}$, we found that the range of $\mathrm{CN}$ strengths does not differ among GCs of different $\mathrm{HB}$ morphologies, as might have been expected in a scenario of internal nucleosynthesis and subsequent dredge-up due to rotationally induced mixing. Thus, stellar rotation could not be clearly identified as a second parameter of horizontal branch morphology. However, the small sample of stars observed in NGC 7006 does not allow us to completely disprove this scenario.

One caveat is necessary to state. If the distribution of angular momentum is a second parameter, it might not necessarily be traced in a sufficient way by $\mathrm{CN}$ variations on the RGB, since an abundance spread due to internal stellar evolution could be hidden by stronger, primordial $\mathrm{CN}$ variations. In fact, recent detections of $\mathrm{CN}$ abundance variations on the faint main-sequence of 47 Tuc strongly suggest that abundance variations due to external pollution effects might exceed the imprint of internal stellar evolution (Harbeck et al. 2003). In so far it is not surprising that we can not link rotationally induced mixing to the problem of the HB morphology in this paper.

A direct measurement of the angular momentum distribution (i.e., the distribution of $v \sin i$ ) in GCs at the main-sequence turn-off (MSTO) is the more appropriate method to investigate the effect of rotation on stellar evolution ${ }^{2}$. In particular, a measurement of the angular momentum distribution would allow a quantitative approach to the connection between rotation and HB morphology. Fiber-fed spectrographs at large telescopes (i.e., FLAMES at the VLT) could allow measurement of rotation speeds of the faint MS stars in nearby globular clusters.

\footnotetext{
2 As bright RGB stars have very extended envelopes, conservation of angular momentum requires extremely low surface rotation speeds of these stars; the broadening of absorption lines in their spectra cannot be used to determine $v \sin i$.
} 
Acknowledgements. GHS gratefully acknowledges the support of NSF grant AST 00-98453.

\section{References}

Behr, B. B., Djorgovski, S. G., Cohen, J. G., et al. 2000, ApJ, 528, 849 Behr, B. B., Cohen, J. G., \& McCarthy, J. K. 2000, ApJ, 531, L37 Briley, M. M., \& Smith, G. H. 1993, in The Globular Cluster-Galaxy Connection, ed. G. H. Smith, \& J. P. Brodie, ASP Conf. Ser., 48, 184

Briley, M. M. 1997, AJ, 114, 1051

Buonanno, R., Pecci, F. F., Capellaro, E., et al. 1991, AJ, 102, 1005

Catelan, M., Bellazzini, M., Landsman, W. B., et al. 2001, AJ, 122, 3171

Cavallo, R. M., Sweigart, A. V., \& Bell, R. A. 1996, ApJ, 464, L79

Cavallo, R. M., Sweigart, A. V., \& Bell, R. A. 1998, ApJ, 492, 575

Cohen, J. G., \& Frogel, J. A. 1982, ApJ, 255, L39

Denisenkov, P. A., \& Denisenkova, S. N. 1990, Soviet Astron. Lett., 16,275

van Dokkum, P. G. 2001, PASP, 113, 1420

Friel, E., Kraft, R. P., Suntzeff, N. B., \& Carbon, D. F. 1982, PASP, 94, 873

Harbeck, D., Grabel, E. K., Holtzman, J., et al. 2001, AJ, 122, 3092

Harbeck, D., Smith, G. H., \& Grebel, E. K. 2003, AJ, 125, 197

Harris, W. E. 1996, AJ, 112, 1487

Hurley-Keller, D., Mateo, M., \& Grebel, E. K. 1999, ApJ, 523, L25

Kraft, R. P. 1994, PASP, 106, 553

Kraft, R. P., Sneden, C., Smith, G. H., Shetrone, M. D., \& Fullbright, J. 1998, AJ, 115, 1500
Langer, G. E., Suntzeff, N. B., \& Kraft, R. P. 1992, PASP, 104, 523

Langer, G. E., Hoffman, R., \& Sneden, C. 1993, PASP, 105, 301

Lee, Y.-W., Demarque, P., \& Zinn, R. 1994, ApJ, 423, 248

McClure, R. D., \& Hesser, J. E. 1981, ApJ, 246, 136

Norris, J., \& Freeman, K. C. 1979, ApJ, 230, L179

Norris, J. 1981, ApJ, 248, 177

Norris, J., Cottrell, P. L., Freeman, K. C., \& Da Costa, G. S. 1981, ApJ, 244, 205

Norris, J., \& Freeman, K. C. 1983, ApJ, 266, 130

Norris, J. 1987, ApJ, 313, 65

Rey, S., Yoon, S., Lee, Y., Chaboyer, B., \& Sarajedini, A. 2001, AJ, 122,3219

Sandage, A., \& Wallerstein, G. 1960, ApJ, 131, 598

Sandage, A., \& Wildey, R. 1967, ApJ, 150, 469

Stetson, P. B., Bolte, M., Harris, W. E., et al. 1999, AJ, 117, 247

Smith, G. H., \& Norris, J. 1982, ApJ, 254, 149

Smith, G. H., \& Bell, R. A. 1986, AJ, 91, 1121

Smith, G. H., \& Mateo, M. 1990, ApJ, 353, 533

Smith, G. H., \& Fullbright, J. 1997, PASP, 109, 1246

Smith, G. H. 2002a, PASP, 114, 1215

Smith, G. H. 2002b, PASP, 114, 1097

Sweigart, A. V. 1997, ApJ, 474, L23

Thoul, A., Jorissen, A., Goriely, S., et al. 2002, A\&A, 383, 491

van den Bergh, S. 1998, ApJ, 495, L79

Wachter, S., Wallerstein, G., Brown, J. A., \& Oke, J. B. 1998, PASP, 110,821

Weiss, A., Denissenkov, P. A., \& Charbonnel, C. 2000, A\&A, 356, 181 